\title{
Metabolism of c-Met Kinase Inhibitors Containing Quinoline by Aldehyde Oxidase, Electron Donating, and Steric Hindrance Effect ${ }^{\mathrm{S}}$
}

\author{
Jiang Wei Zhang, Wen Xiao, Zhen Ting Gao, Zheng Tian Yu, and Ji Yue (Jeff) Zhang \\ China Novartis Institutes for BioMedical Research, Shanghai, People's Republic of China
}

Received April 26, 2018; accepted September 10, 2018

\begin{abstract}
Some quinoline-containing c-Met kinase inhibitors are aldehyde oxidase (AO) substrates. 3-Substituted quinoline triazolopyridine analogs were synthesized to understand the electron-donating and steric hindrance effects on AO-mediated metabolism. Metabolic stability studies for these quinoline analogs were carried out in liver cytosol from mice, rats, cynomolgus monkeys, and humans. Several 3-N-substituted analogs were found to be unstable in monkey liver cytosolic incubations (half-life, $<10$ minutes), and five of them $(63,53,51,11$, and 71$)$ were chosen for additional mechanistic studies. Mono-oxygenation on the quinoline ring was identified by liquid chromatography tandem mass spectrometry. Metabolite formation was inhibited by the $A O$ inhibitors menadione and raloxifene, but not by the xanthine oxidase inhibitor
\end{abstract}

\section{Introduction}

c-Met is a receptor tyrosine kinase for hepatocyte growth factor. c-Met overexpression and mutation are associated with tumorigenesis, metastasis, poor prognosis, and drug resistance (Cañadas et al., 2010; Liu et al., 2010; Parikh and Ghate, 2018). c-Met has become an attractive target for non-small cell lung cancer and other advanced cancers, resulting in many small-molecule c-Met inhibitors in clinical trials (Fig. 1) (Cañadas et al., 2010; Liu et al., 2010; Cui et al., 2013; Cui, 2014).

Aldehyde oxidase (AO) plays an important role in the metabolism of aromatic aza-heterocycles (Kitamura et al., 2006; Pryde et al., 2010). As an aza-heterocycle, quinoline is an essential binding motif in class Ib selective c-Met inhibitors, such as capmatinib, OMO-1, AMG-208 (7-methoxy-4-[(6phenyl[1,2,4]triazolo[4,3-b]pyridazin-3-yl)methoxy]quinoline), SGX523 [6(6-(1-methyl-1H-pyrazol-4-yl)-[1,2,4]triazolo[4,3-b]pyridazin-3-ylthio)quinolone], JNJ-38877605 [6-(difluoro(6-(1-methyl-1H-pyrazol-4-yl)[1,2,4]triazolo[4,3-b]pyridazin-3-yl)methyl)quinolone], and PF-04217903 [2-(4-(3-quinolin-6-ylmethyl-3H-(1,2,3)triazolo(4,5-b)pyrazin-5-yl)pyrazol1-yl)ethanol] (Fig. 1) (Cui, 2014). Many quinolone-containing c-Met kinase inhibitors were reported to be AO substrates, including SGX523 and JNJ-38877605 (Diamond et al., 2010; Lolkema et al., 2015). The C-H bond at the 2-quinoline position was metabolized by human $\mathrm{AO}$ to form a lactam metabolite (M) (Kitamura et al., 2006; Pryde et al., 2010). Being less soluble and prone to crystallize in the kidney, the lactam $\mathrm{M}$ was

https://doi.org/10.1124/dmd.118.081919.

S This article has supplemental material available at dmd.aspetjournals.org. allopurinol. It was found that small electron-donating groups at the 3-quinoline moiety made the analogs more susceptible to $\mathrm{AO}$ metabolism, whereas large 3-substituents could reverse the trend. Although species differences were observed, this trend was applicable to all species tested. Small electron-donating substituents at the 3-quinoline moiety increased both affinity (decreased Michaelis constant) and $V_{\max }$ maximum velocity toward $A O$ in kinetic studies, whereas large substituents decreased both parameters probably as a result of steric hindrance. Based on our analysis, a common structural feature with high $\mathrm{AO}$ liability was proposed. Our finding could provide useful information for chemists to minimize potential $\mathrm{AO}$ liability when designing quinoline analogs. believed to be the culprit of nephrotoxicity and ultimately clinical failure for SGX523 and JNJ-38877605 (Diamond et al., 2010; Lolkema et al., 2015). To overcome the AO metabolism-related low-solubility issue, some efforts have been made to modify the quinoline moiety, such as converting it to an imidazopyridine moiety in volitinib (Jia et al., 2014) or replacing it with an isoquinolinone moiety (Ryu et al., 2011). In addition, no AO liability was observed when some bulky substituents were added at the 3-quinoline (Zhao et al., 2017). We have discovered a series of triazolopyridine analogs to be potent c-Met inhibitors and have attempted to add $\mathrm{N}$-substituents at the 3-quinoline to block or reduce AO-mediated metabolism (Chen et al., 2013). However, several of these substituted analogs were found to be unstable in a cytosolic stability assay, suggesting that they were still liable for AO-mediated metabolism.

This study was aimed to evaluate the relationship between $\mathrm{AO}$ metabolism and quinoline with different $\mathrm{N}$-substituents at the 3-position for triazolopyridine c-Met inhibitors. Electron-donating groups (EDGs) and steric hindrance were found to affect $\mathrm{AO}$ metabolism. Our findings could provide useful information for chemists to minimize potential $\mathrm{AO}$ liability when designing quinoline analogs.

\section{Materials and Methods}

Chemicals. Triazolopyridine compounds, SGX523, and INC280 (2-fluoro-Nmethyl-4-[7-(quinolin-6-ylmethyl)imidazo[1,2-b][1,2,4]triazin-2-yl]benzamide) were synthesized at China Novartis Institute of Biomedical Research (Shanghai, People's Republic of China) (Chen et al., 2013). Allopurinol and menadione were purchased from Sigma-Aldrich (St. Louis, MO). Raloxifene was purchased from

ABBREVIATIONS: ACN, acetonitrile; AO, aldehyde oxidase; c-Met, mesenchymal-epithelial transition factor; DMSO, dimethylsulfoxide; EDG, electron-donating group; $\Delta \mathrm{G}$, energy needed to form tetrahedral intermediate from substrate and $\mathrm{H}_{2} \mathrm{O}$; $K_{\mathrm{m}}$, Michaelis constant; LC, liquid chromatography; M, metabolite; MRM, multiple reaction monitoring; MS, mass spectrometry; MS/MS, tandem mass spectrometry; $\mathrm{P}$, parent; $t_{1 / 2}$, half-life; $\mathrm{XO}$, xanthine oxidase. 
<smiles></smiles>

Savolitinib

Phase III<smiles>COCCOc1cnc2cn([C@@H](C)c3nnc4c(F)cc(-c5cnn(C)c5)cn34)c(=O)cc2c1</smiles>

AMG-337

Phase II<smiles>COc1ccc2c(OCc3nnc4ccc(-c5ccccc5)nn34)ccnc2c1</smiles>

AMG-208

Phase I discontinued<smiles>COC(=O)OCc1ccc2ncccc2c1</smiles>

SGX523

Phase I terminated<smiles>CNC(=O)c1ccc(-c2cnc3ncc(Cc4ccc5ncccc5c4)n3n2)cc1F</smiles>

Capmatinib<smiles>FC(F)(c1ccc2ncccc2c1)c1nnc2ccc(-c3ccncc3)nn12</smiles>

OMO-1
Phase II<smiles>Cc1ccc2cc(C(F)(F)c3nnc4ccc(-c5cnn(C)c5)nn34)ccc2n1</smiles>

JNJ-38877605
Fig. 1. c-Met inhibitors in clinical trials with quinoline or quinoline mimics. Asterisks indicate an $\mathrm{AO}$ metabolism site.
Shanghai DEMO Chemical Co. Ltd. (Shanghai, People's Republic of China). Pooled mouse liver cytosol (male CD1, $n=1000$ ) and pooled rat liver cytosol (male IGS SD, $n=400$ ) were purchased from Xenotech (Lenexa, KS). Pooled monkey liver cytosol (male cynomolgus) was purchased from IPhase Pharma Service (Beijing, People's Republic of China). Pooled human liver cytosol (Ultrapool 150) was purchased from BD Biosciences (San Jose, CA). All other reagents were of high-performance liquid chromatography grade or of analytical grade.

Metabolic Stability. Parent (P) compound depletion in cytosolic incubation was used to measure metabolic stability. The incubation, with a total volume of $200 \mu$ l, was conducted in $50 \mathrm{mM}$ potassium phosphate buffer ( $\mathrm{pH}$ 7.4) with liver cytosol $(2 \mathrm{mg} / \mathrm{ml})$ and test compounds $(1 \mu \mathrm{M})$. Test compounds were dissolved in dimethylsulfoxide (DMSO) to $10 \mathrm{mM}$, then further diluted in water to $2 \mu \mathrm{M}$. The final concentration of DMSO was $<0.1 \%$ for all incubations. After 15 minutes of preincubation at $37^{\circ} \mathrm{C}$, the reaction was initiated by adding the test compound. After $0,30,60$, and 120 minutes of incubation at $37^{\circ} \mathrm{C}, 40-\mu 1$ aliquots were removed and mixed with $100 \mu \mathrm{l}$ of acetonitrile $(\mathrm{ACN})$ containing internal standard to terminate the reaction. The mixture was centrifuged at $3400 \mathrm{~g}$ for 10 minutes at room temperature. The supernatants $(50 \mu \mathrm{l})$ were diluted with Milli-Q water $(50 \mu \mathrm{l})$ and analyzed by liquid chromatography mass spectrometry (LC-MS). The mass response of the $\mathrm{P}$ compound at 0 minute was set at $100 \%$. The rate constant of substrate depletion, $k$, was the slope of the semi-logarithmic plot of the percentage remaining versus time. The in vitro half-life $\left(t_{1 / 2}\right)$ was calculated using the following equation: $t_{1 / 2}=\ln 2 / k$.

The LC-tandem MS (LC-MS/MS) system consisted of the Shimadzu (Kyoto, Japan) UFLC LC20A System, an AB Sciex (Framingham, MA) API5000 Mass Spectrometer, and a Chromolith FastGradient C18 column $(2.0 \times 50 \mathrm{~mm}$; Merck
$\mathrm{KGaA}$, Darmstadt, Germany). Mobile phases A and $\mathrm{B}$ were $\mathrm{H}_{2} \mathrm{O}$ and $\mathrm{ACN}$, respectively, with $0.1 \%$ formic acid as an additive in both solvents. Mobile phase B was increased from $2 \%$ to $80 \%$ over 0.3 minute, and was kept at $80 \%$ for another 0.4 minute before being changed back to $2 \%$. The flow rate was $600 \mu \mathrm{l} / \mathrm{min}$ with a total run time of 2 minutes. The ionization was optimized using atmospheric pressure chemical ionization $(+)$, and the detection was performed via multiple reaction monitoring (MRM). Other mass parameters were as follows: collision gas at $6 \mathrm{psi}$, curtain gas at $35 \mathrm{psi}$, ion source gas 1 at $50 \mathrm{psi}$, temperature at $450^{\circ} \mathrm{C}$, nebulizer current at $3 \mathrm{~V}$, entrance potential at $10 \mathrm{~V}$, and collision cell exit potential at $15 \mathrm{~V}$.

M Identification. The incubation system was similar to that mentioned above in the metabolic stability study. Briefly, in the incubation system with a total volume of $200 \mu \mathrm{l}$, the test compound $(5 \mu \mathrm{M})$ was incubated with $1.0 \mathrm{mg} / \mathrm{ml}$ monkey liver cytosol in potassium phosphate buffer $(\mathrm{pH} 7.4,50 \mathrm{mM})$ at $37^{\circ} \mathrm{C}$ for 120 minutes. The reaction was terminated by adding $400 \mu \mathrm{l}$ of ACN. After centrifugation, the supernatant was then transferred into a new vial and evaporated to dryness in vacuo. The residue was reconstituted with $100 \mu \mathrm{l}$ of $50 \% \mathrm{ACN}$ in $\mathrm{H}_{2} \mathrm{O}$ and subjected to direct LC-MS/MS analysis. The LC-MS/MS system was a Thermo Accela high-performance liquid chromatography coupled with a Thermo LTQ OrbriTrap XL ion-trap mass spectrometer (Thermo Fisher Scientific, Waltham, MA) and an Agilent XDB- ${ }_{18}$ column $(4.6 \times 50 \mathrm{~mm}, 1.8 \mu \mathrm{m}$; Agilent Technologies, Santa Clara, CA). The mobile phase A was $0.1 \%$ formic acid in water, and mobile phase B was $0.1 \%$ formic acid in ACN. The mobile phase B was increased from $2 \%$ B to $98 \%$ B over 20 minutes using a flow rate of $300 \mu 1 / \mathrm{min}$. The mass spectrometer was operated in a positive ion mode with an electrospray ionization source. Samples were analyzed using a linear ion trap Q1 scan with information-dependent acquisition-triggered product ion (MS2) scans. The 
TABLE 1

Kinetic study conditions for triazolopyridine compounds in liver cytosol incubation from different species

\begin{tabular}{llccccc}
\hline & & \multicolumn{5}{c}{ Test Compounds } \\
\cline { 3 - 6 } & Species & 71 & 11 & 51 & 53 & 63 \\
\hline Compound concentration $(\mu \mathrm{M})$ & & $0.23-30$ & $0.47-60$ & $0.23-30$ & $0.23-30$ & $0.47-60$ \\
Liver cytosol protein concentration $(\mathrm{mg} / \mathrm{ml})$ & Monkey & 0.1 & 0.2 & 0.1 & 0.1 & 1.0 \\
& Mouse & 0.5 & 0.5 & 0.5 & 0.5 & 1.0 \\
& Human & 0.5 & 0.5 & 0.5 & 0.5 & 1.0 \\
& Rat & 0.5 & 0.5 & 0.5 & 0.5 & 1.0 \\
\hline
\end{tabular}

following detailed instrument settings were listed: capillary temperature at $275^{\circ} \mathrm{C}$; sheath gas flow at $10 \mathrm{psi}$; source voltage at $4.5 \mathrm{kV}$; and capillary voltage at $24 \mathrm{~V}$.

Chemical Inhibition. Compounds $63,53,51,11$, and 71 were selected for chemical inhibition study in human liver cytosol because of their high metabolism conversion rates. Chemical inhibition studies were performed by adding various molybdenum hydroxylase inhibitors to identify the enzyme responsible for the metabolism. In a total 50- $\mu$ l incubation system, each compound (5 $\mu \mathrm{M})$ was incubated with human liver cytosol $(0.5 \mathrm{mg} / \mathrm{ml})$ for 30 minutes. Various molybdenum hydroxylase inhibitors and their final concentrations were listed

TABLE 2

Structure, $t_{1 / 2}$, molecular volume, $\Delta \mathrm{G}$, and docking score for the 3 -substituted quinoline analogs

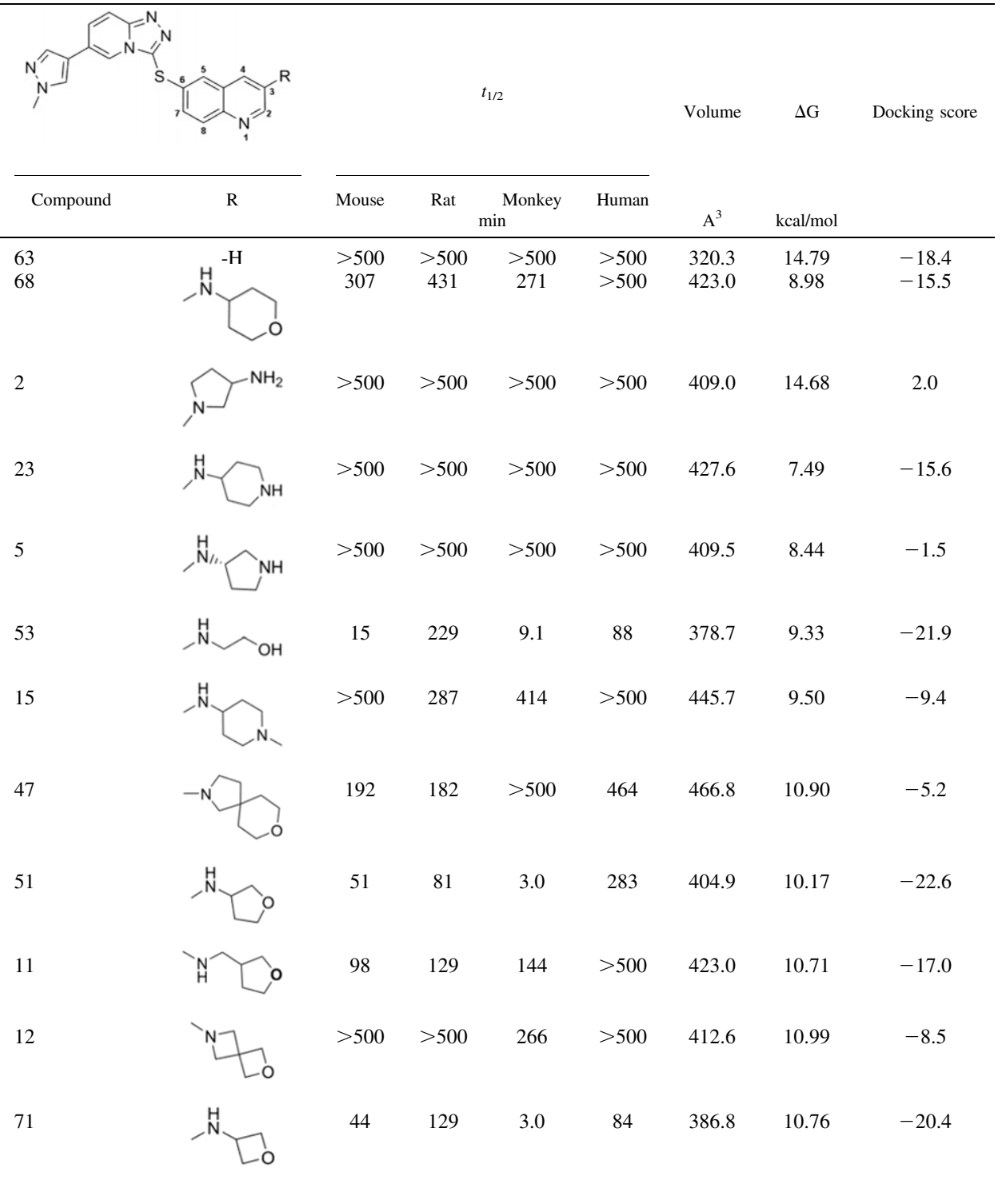

$\mathrm{T}_{1 / 2}$ values were determined with substrate concentration of $1 \mu \mathrm{M}$ and liver cytosol protein concentration of $2 \mathrm{mg} / \mathrm{ml}$. 
as follows: menadione (50 $\mu \mathrm{M})$ (Johns, 1967), raloxifene (20 $\mu \mathrm{M})$ (Obach, 2004), and allopurinol $(50 \mu \mathrm{M})$ (Massey et al., 1970). These chemical inhibitors were dissolved in DMSO and diluted with water to produce working solutions. For all of the experiments, the final concentration of DMSO was less than $0.15 \%(\mathrm{v} / \mathrm{v})$. In the controls, an equal volume of solvent was added. There was 10 minutes of preincubation, and the reaction was initiated by the addition of the substrate. The termination, centrifugation, and dilution steps were the same as in the metabolic stability study. A $5 \mu \mathrm{M}$ concentration was selected around the $K_{\mathrm{m}}$ value so that detectable $\mathrm{M}$ could be produced. Oxidative metabolites were detected by LC-MS using predictive MRM methods derived from their corresponding P compounds. The predicted MRMs for oxidative Ms and their P compounds were listed as follows: 63 (P, $359 \rightarrow$ 173; M, $375 \rightarrow$ 173); 53 (P, $418 \rightarrow$ 146; M, $434 \rightarrow 146$ ); $51(\mathrm{P}, 444 \rightarrow 146 ; \mathrm{M}, 460 \rightarrow 146) ; 11(\mathrm{P}, 458 \rightarrow 173 ; \mathrm{M}, 474 \rightarrow 173)$; and $71(\mathrm{P}$, $430 \rightarrow 400 ; \mathrm{M}, 446 \rightarrow 416$ ). All incubations were performed in duplicate unless stated otherwise.

Kinetic Studies. To estimate kinetic parameters, test compounds were incubated with liver cytosol from different species for 30 minutes. The incubation conditions such as substrate and cytosol concentrations were listed in Table 1. The apparent $V_{\max }$ and $K_{\mathrm{m}}$ values were calculated by nonlinear regression analysis of experimental data according to the Michaelis-Menten equation. Preliminary experiments were performed to make sure that formations of Ms were in the linear range for both reaction time and cytosol concentration. The Ms were measured using an MRM mode. Due to the lack of M standards, the quantifications were based on the calibration curves from corresponding parent compounds (5-1000 $\mathrm{nM}$ ), assuming similar a mass response. All incubations were performed in duplicate with S.D. values generally below $10 \%$.

In Silico Methods. During AO catalyzed oxidation process, the formation of a tetrahedral intermediate (-CHOH-NH-) was hypothesized by adding $\mathrm{H}_{2} \mathrm{O}$ to the substrate on the $-\mathrm{C}=\mathrm{N}$ - double bond (Torres et al., 2007; Dalvie et al., 2012; Xu et al., 2017). The energy required to form the tetrahedral intermediate $(\Delta G)$ is defined as follows: $\Delta G=G_{\text {inter }}-G_{\text {sub }}-G_{H 2 O}$, where $G_{\text {inter }}, G_{\text {sub }}$, and $G_{H 2 O}$ represent the single point energy of intermediate, substrate, and water, respectively. Single point energy was calculated according to the methods reported (Torres et al., 2007; Dalvie et al., 2012; $\mathrm{Xu}$ et al., 2017) with slight modifications. Briefly, 3D structures were first optimized using Spartan'06 (Wavefunction Inc., Irvine, CA) using the minimization function. Then single point energy was determined by Spartan using the B3LYP/6-31G** basis set. No solvent model was considered during the calculation.

Docking Studies. To evaluate the steric hindrance effect, molecular volume was calculated and ligands were docked into the active site of AO receptor (PDB entry 4uhx) (Coelho et al., 2015). Focus 3.83 software (Molsoft L.L.C., San Diego, CA) was used for molecular volume calculation and docking studies (Stiefl et al., 2015). Both the ligands and the receptor were optimized in Focus before docking. A $40 \times 40 \times 40$ point grid centered on phthalazine was used as a docking region. The productive pose with the lowest docking score was used for analysis.

\section{Results}

Metabolic Stability. The metabolic stability of quinoline analogs was carried out in liver cytosol isolated from mice, rats, cynomolgus monkeys, and humans (Table 2). SGX523 and INC280 were included as controls, and the $t_{1 / 2}$ values are listed in Supplemental Table 1. Compound 63 had no substituent on the quinoline moiety and showed slow turnover in liver cytosolic incubation with $t_{1 / 2}$ values $>500$ minutes in all four species tested. Some small $\mathrm{N}$-substituents at the 3-position made the compounds unstable. $t_{1 / 2}$ values of less than 10 minutes were observed for compounds 53, 51, and 71 in monkey liver cytosolic incubations (Table 2). Similarly, these compounds were also unstable in liver cytosolic incubation from mice, rats, and humans. Based on the $t_{1 / 2}$ values, AO activity was the highest in monkeys, followed by mice, and the lowest in rats and humans for compounds $68,53,51$, and 71 . This rank of order was not applicable to compounds 11,47 , and 15 . Therefore, species difference seemed to be compound specific, and there was no constant pattern (Sahi et al., 2008).
As the size of the $\mathrm{N}$-substituent increased, the compounds became more stable (longer $t_{1 / 2}$ ). $t_{1 / 2}$ values of $>2$ hours were observed in all four species for compounds $68,2,23,5,15,47$, and 12. Compared with compound 51, compound 11 had only one extra methylene linker and its $t_{1 / 2}$ value was much longer (144 vs. 3 minutes in the monkey). The lowest molecule volumes were observed for compounds 53, 51, and 71 , corresponding to the least stable compounds in the list (Table 2). These observations showed that small EDG substituents increased AO-mediated metabolism, while large substituents had an opposite effect probably due to steric hindrance.

M Identification. The least stable compounds (53, 51, 11, and 71) were chosen for $\mathrm{M}$ identification study in monkey liver cytosol. Compound 63 was included for the comparison. Mono-oxygenated Ms $(m / z+16)$ with a longer LC retention time than parent compounds were observed for these compounds using LC-MS (Fig. 2). The yields were $83 \%$ (compound 71), 75\% (compound 51), 57\% (compound 53), $13 \%$ (compound 11), and $3 \%$ (compound 63) after 2 hours of incubation. Compared with 63, N-substituents at the 3-position of quinoline moiety made compounds less stable in cynomolgus monkey liver cytosol, as the yield increased. A large $\mathrm{N}$-substituent at the 3-position (11) reduced the yield, indicating the steric hindrance effect. This finding is consistent with the results obtained in a metabolic stability study.

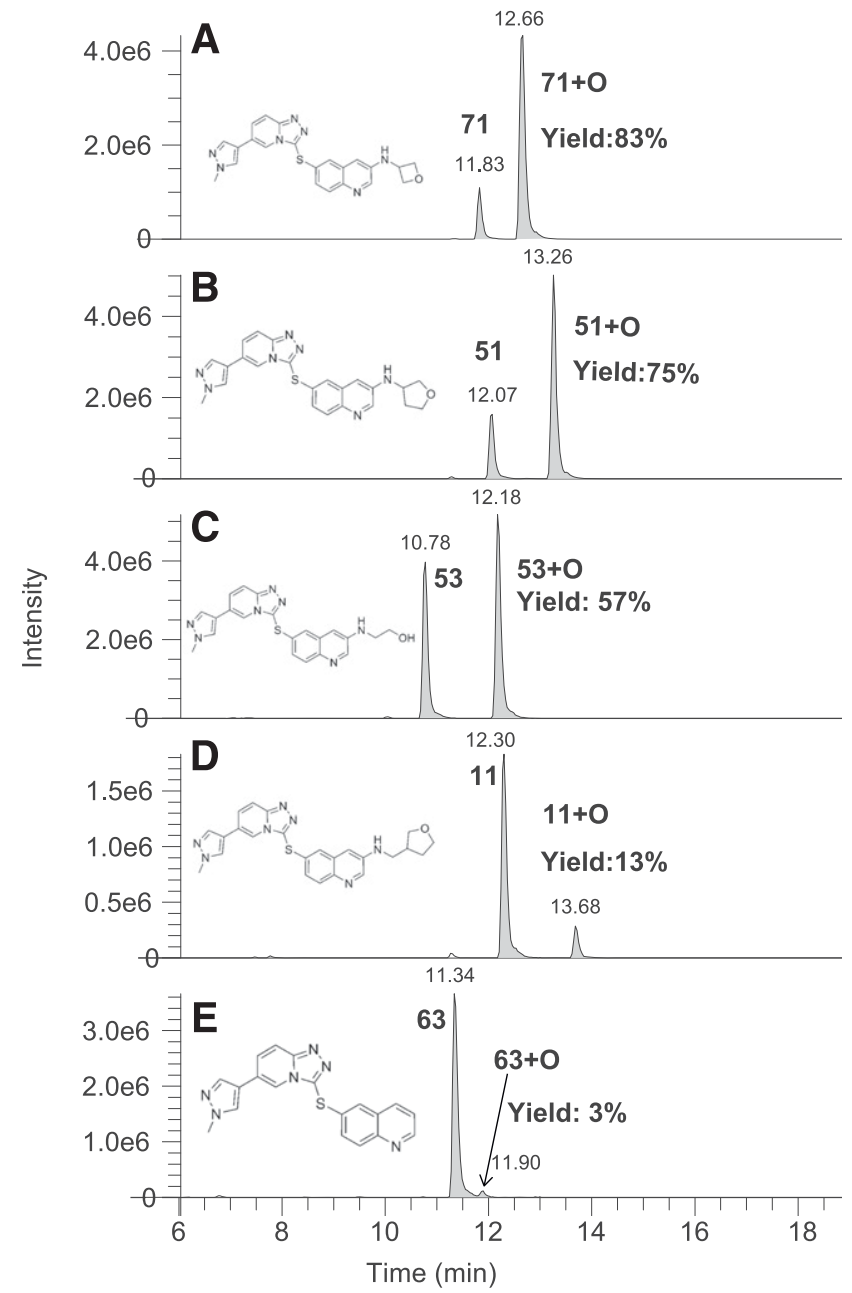

Fig. 2. Ion extraction chromatography for selected triazolopyridine analogs (A:71; $\mathrm{B}: 51$; C:53; D:11; E:63) and their corresponding mono-oxygenated Ms. Yield is defined as the substrate conversion after a test compound $(5 \mu \mathrm{M})$ was incubated with monkey liver cytosol $(1 \mathrm{mg} / \mathrm{ml})$ for 2 hours. 
TABLE 3

LC-MS/MS fragments of $\mathrm{P}$ compounds and their respective oxidative Ms

Mass Fragments
[M+H]

The MS/MS fragments of parent compounds and their proposed Ms are listed in Supplemental Fig. 1 and Table 3. The oxidation was proposed to occur on the quinoline part of the molecule based on MS/MS fragmentation. Many studies showed that the vacant $\mathrm{C}-\mathrm{H}$ bond adjacent to the nitrogen atom in an aromatic heterocycle was metabolized to form a lactam M by molybdenum hydroxylase (Kitamura et al., 2006; Pryde et al., 2010). The 2-quinoline was assumed to be oxidized to yield a lactam accordingly (Table 3 ). The structure assignment was consistent with a longer retention time of a 2-oxo-quinoline $\mathrm{M}$ compared with the parent compound observed for quinoline containing c-Met inhibitors such as SGX523, capmatinib, and many others (Diamond et al., 2010; Xu et al., 2017; Dick, 2018). No further study was performed to confirm the $\mathrm{M}$ structures.

Chemical Inhibition. Molybdenum hydroxylase was involved in the oxidative metabolism of these quinoline analogs in cytosol without NADPH. To identify the enzyme involved in the metabolism, molybdenum hydroxylase inhibitors were added to the incubation system. Allopurinol (XO inhibitor) exhibited limited or no inhibition $(<17 \%)$ toward all of the analogs tested. Both menadione and raloxifene (AO inhibitors) inhibited over $75 \%$ of the $\mathrm{M}$ formation of compounds 63, 53, 51, and 71 (Fig. 3; Table 4). For compound 11, the $\mathrm{M}$ formation was inhibited by $51 \%$ (menadione) and $35 \%$ (raloxifene) in human liver cytosolic incubation. Relatively low inhibition of compound 11 in human liver cytosolic incubation was probably due to its low conversion rate. Therefore, an inhibition study was repeated in mouse liver cytosol, in which a high conversion rate was observed for compound 11 . The metabolism of compound 11 was inhibited by $93 \%$ and $86 \%$ by menadione and raloxifene, respectively. These data revealed that $\mathrm{AO}$, and not $\mathrm{XO}$, was the enzyme responsible for the quinoline metabolism.

Kinetic Study. To further explain the observed differences in the metabolism of quinoline analogs, kinetic studies were performed in four species (Fig. 4; Table 5). In agreement with the $\mathrm{M}$ identification study, compound 63 had the lowest clearance judging from the $V_{\text {max }} / K_{\mathrm{m}}$ value $(<0.5 \mu \mathrm{l} / \mathrm{min}$ per milligram). The $\mathrm{N}$-substituent at the 3-position of the quinoline moiety increased AO metabolism (increased $V_{\max } / K_{\mathrm{m}}$ value). Compared with compound 63, decreased $K_{\mathrm{m}}$ values were observed for all $\mathrm{N}$-substituent analogs in all species, indicating increased binding affinity. Compared with compound 63 , increased $V_{\max }$ values were observed for all compounds in all species except for compounds 11 and 51 in human liver cytosol (Fig. 4; Table 5). Compound 11 had the lowest $V_{\max } /$ $K_{\mathrm{m}}$ value among $\mathrm{N}$-substituent analogs, again suggesting that large $\mathrm{N}$-substituents impede AO metabolism by steric hindrance. Species differences were also observed. Cynomolgus monkeys had the largest $V_{\max }$ value for compounds 71, 11, and 51; whereas the $V_{\max } /$ $K_{\mathrm{m}}$ value was the highest in mice for compounds $11,51,53$, and 63 . The lowest $V_{\max } / K_{\mathrm{m}}$ value was observed in humans for compounds 71,11 , and 51 .

\section{Discussion}

There are many c-Met inhibitors containing a quinoline moiety in the clinical trials (Fig. 1) (Cui, 2014), and AO has been reported to play a 

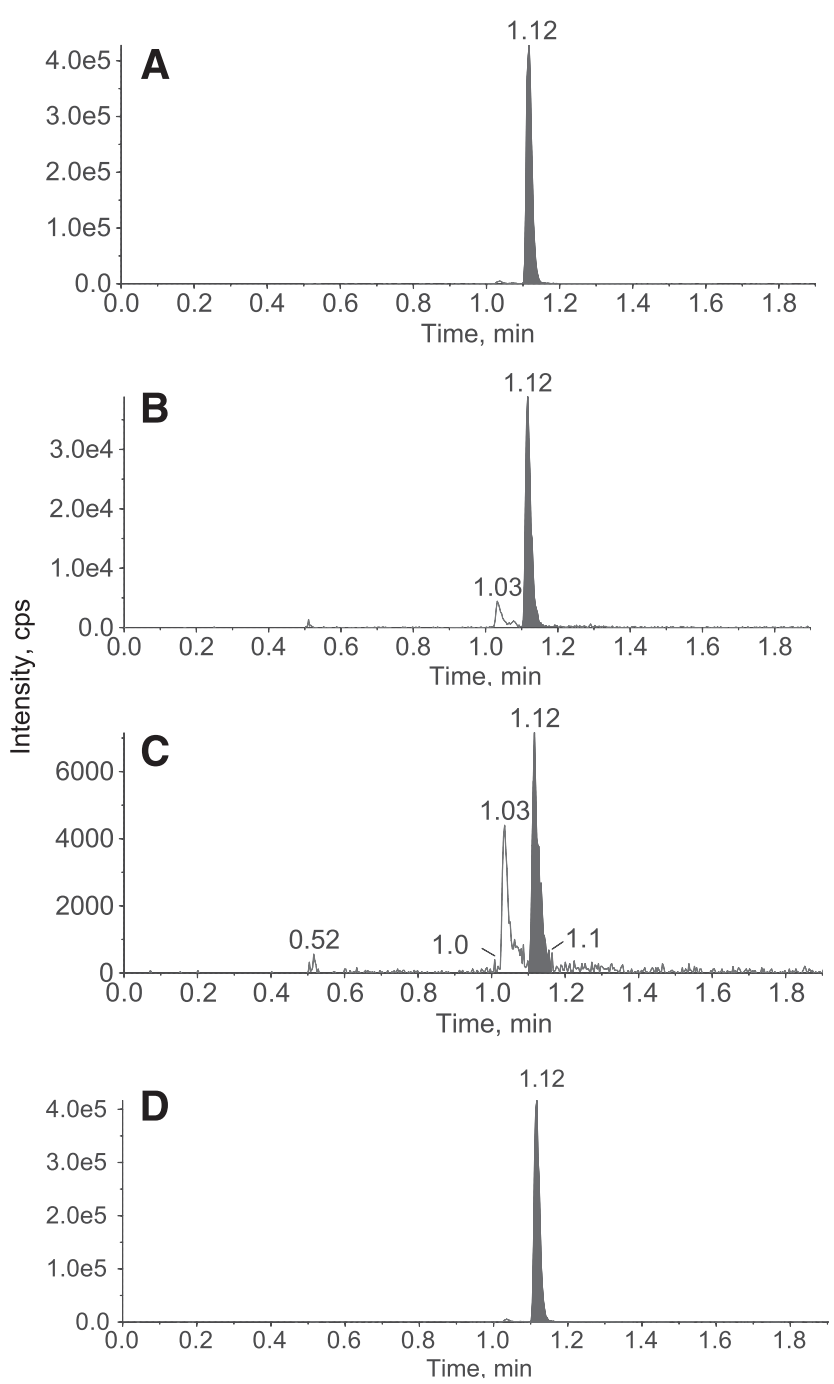

Fig. 3. Representative LC-MS in a chemical inhibition study. The AO $\mathrm{M}$ of compound 53 appears at 1.12 minutes. The following inhibitors were used: solvent control (A); menadione (50 $\mu \mathrm{M})(\mathrm{B})$; raloxifene $(20 \mu \mathrm{M})(\mathrm{C})$; and allopurinol $(50 \mu \mathrm{M})(\mathrm{D})$.

major role in the quinoline metabolism (Xu et al., 2017). The clinical failure of SGX523 and PF-04217903 was reported due to the low solubility of their AO-mediated Ms, which precipitated in the kidney and led to renal failure (Diamond et al., 2010; Lolkema et al., 2015). A novel series of 3-N-substituted quinoline triazolopyridine analogs was discovered as potent c-Met inhibitors with a clean PDE3 profile (Chen et al., 2013). To monitor AO-mediated metabolism, AO liability was routinely screened in monkey liver cytosol. In theory, extra substituents should increase steric hindrance to deter AO metabolism. However, several 3-substituted quinoline analogs were found to be less stable than the unsubstituted quinoline. This prompted us to investigate the mechanism.

In the present study, cytosol was used to measure AO metabolism. The selection of cytosol would exclude other metabolizing enzymes or transporters and simplify the system to consider cytosolic enzymes only. The site of metabolism was ascribed to the quinoline moiety based on the MS/MS fragmentation and the characteristic longer retention time compared with the parent compound. An additional chemical inhibition study revealed that $\mathrm{AO}$, but not $\mathrm{XO}$, was the principal metabolizing enzyme. Small electron-donating $\mathrm{N}$-substituents on the 3-quinoline increased $\mathrm{AO}$ metabolism, whereas bulky $\mathrm{N}$-substituents would exhibit the steric hindrance effect. The EDG and steric hindrance effects observed in this study were in agreement with the findings of these reported studies (Jones and Korzekwa, 2013; Lepri et al., 2017; Xu et al., 2017).

Molybdenum hydroxylases catalyzed oxidation by nucleophilic attack at the carbon atom adjacent to the nitrogen in aromatic heterocycles (Pryde et al., 2010). The energy needed to form the tetrahedral intermediate $(\Delta \mathrm{G})$ was used to predict $\mathrm{AO}$ metabolism (Torres et al., 2007). And a low $\Delta \mathrm{G}$ value favors $\mathrm{AO}$ metabolism (shorter $\left.t_{1 / 2}\right) . \Delta \mathrm{G}$ values were calculated, and the results are shown in Table 2. Lower $\Delta \mathrm{G}$ values were observed for all $3-\mathrm{N}$-substituted quinoline compounds. $\Delta \mathrm{G}$ values of at least $4 \mathrm{kcal} / \mathrm{mol}$ lower than that for compound 63 were observed for the high-clearance compounds 53, 51, 11 and 71 . However, low $\Delta \mathrm{G}$ values were also observed for compounds $68,23,5$, and $15(<10.7 \mathrm{kcal} / \mathrm{mol})$, which are rather stable compounds. These observations suggest that the $\Delta \mathrm{G}$ value alone cannot predict AO stability.

In addition to $\Delta \mathrm{G}$, steric hindrance was reported as another main factor affecting AO metabolism (Torres et al., 2007; Dalvie et al., 2012; Lepri et al., 2017; Xu et al., 2017). To evaluate the steric hindrance effect, these analogs were docked to the active site of AO (Protein Data Bank entry 4UHX) (Coelho et al., 2015), and docking scores are listed in Table 2. A docking score can reflect the steric hindrance effect in ligand binding, and a lower docking score favors the reaction. A docking score more than $2 \mathrm{U}$ lower was observed for good AO substrates such as compounds 53, 51, and 71. Low docking scores were probably due to the extra hydrogen bond formation. The tetrahydrofuran or oxetane moiety in compounds 51 and 71 provided a hydrogen bond acceptor for residue G1019, and the hydroxyl group in compound 53 supplied both a hydrogen bond acceptor and a donor for residues G1017 and G1088, respectively. Higher docking scores were observed for other analogs. The $t_{1 / 2}$ difference between two matched pairs ( 51 vs. 5 and 68 vs. 23) probably implied the hydrogen bond donor was not well tolerated. Primary and secondary amine analogs (compounds 2, 23, and 5) were stable in cytosol (Table 2).

Many 4- or 5-linked quinoline compounds were AO substrates with high clearance and short $t_{1 / 2}$ in cytosol, such as ML-347 (5-[6-(4-

TABLE 4

Inhibition of quinoline analogs metabolism by various molybdenum hydroxylase inhibitors in liver cytosol fractions

\begin{tabular}{lcrrrrrr}
\hline & \multirow{2}{*}{ Species } & \multicolumn{5}{c}{ Percentage of Control Activity } \\
\cline { 3 - 7 } & & Human & Human & Human & Human & Human & Mouse \\
\hline & & & & & $\%$ & & \\
Inhibitor (concentration) & Analog & 63 & 53 & 51 & 11 & 71 & 11 \\
Menadione $(50 \mu \mathrm{M})$ & & 20 & 9 & 22 & 49 & 9 & 7 \\
Raloxifene $(20 \mu \mathrm{M})$ & & 23 & 2 & 13 & 65 & 2 & 14 \\
Allopurinol $(50 \mu \mathrm{M})$ & & 118 & 83 & 92 & 110 & 86 & 98 \\
\hline
\end{tabular}


methoxyphenyl)pyrazolo[1,5-a]pyrimidin-3-yl]-quinoline), LDN-193189 (4-[6-[4-(1-piperazinyl)phenyl]pyrazolo[1,5-a]pyrimidin-3-yl]-quinoline), A-77-01 (4-[3-(6-methyl-2-pyridinyl)-1H-pyrazol-4-yl]-quinoline), zoniporide, and SB-277011 (N-[trans-4-[2-(6-cyano-3,4-dihydro-2 $(1 \mathrm{H})-$ isoquinolinyl)ethyl]cyclohexyl]-4-quinolinecarboxamide (Austin et al., 2001; Dalvie et al., 2012; Dick, 2018). However, 6-linked quinoline c-Met inhibitors such as SGX523 (Diamond et al., 2010), PF-04217903 (Zientek et al., 2010; Xu et al., 2017), BVU972 (6-[[6-(1-methylpyrazol4-yl)imidazo[1,2-b]pyridazin-3-yl]methyl]quinoline) (Xu et al., 2017), and INC280 (Xu et al., 2017; Dick, 2018) were more stable. Compound 63 is an AO substrate, but, like those 6-linked quinoline c-Met inhibitors, its AO metabolism rate was slow $\left(t_{1 / 2}>2\right.$ hours $)$ in cytosolic incubations from both rodents and primates (Table 2 ). These data could explain why so many 6-linked quinoline c-Met inhibitors are in the clinical trials despite AO-mediated metabolism. The $\Delta \mathrm{G}$ values (in $\mathrm{kcal} / \mathrm{mol}$ ) for these 4 - or 5-linked quinoline compounds were calculated to be 13.4 (ML-347), 15.8 (LDN-193189), 20.5 (A77-01), 10.6 (zoniporide), and 19.3 (SB-277011). The low $\Delta \mathrm{G}$ value could explain the short $t_{1 / 2}$ for zoniporide. The $\Delta \mathrm{G}$ values for ML-347, LDN-193189, A-77-01, and SB-277011 were comparable or even higher than that for compound 63. Therefore, their high AO-mediated metabolism rates were not caused by an electron effect such as $\Delta \mathrm{G}$. These observations may suggest the quinoline linkage positions exert a different steric hindrance effect and the 6-linkage is generally more resistant to AO metabolism than the 4- or 5-linkage.
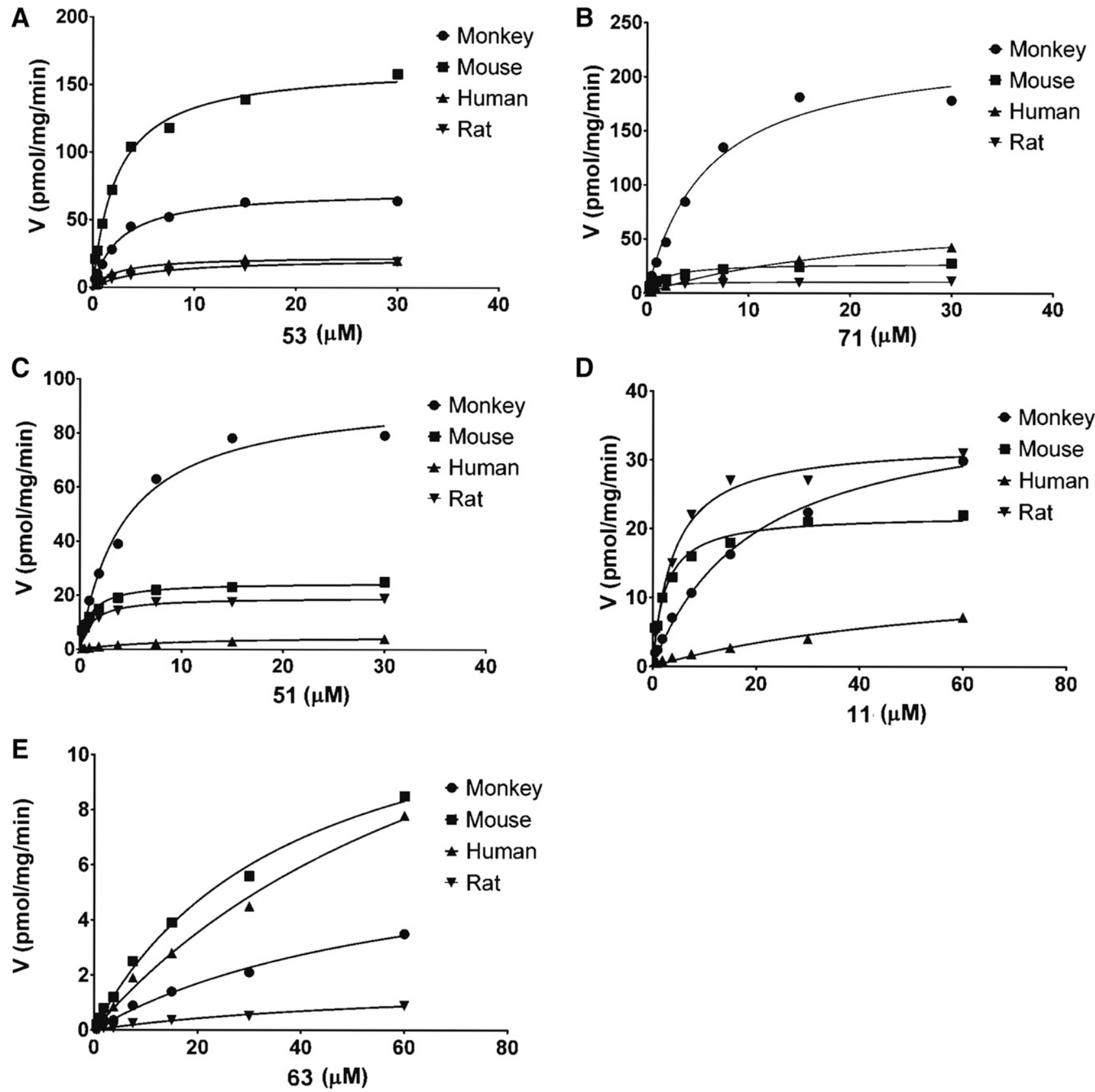

Fig. 4. Michaelis-Menten plot of quinoline analog (A:53; B:71; C:51; D:11; E:63) metabolism in liver cytosol fractions from different species. Each triazolopyridine analog $(0.23-60 \mu \mathrm{M})$ was incubated with liver cytosol fractions $(0.1-1 \mathrm{mg} / \mathrm{ml})$ for 30 minutes. 
TABLE 5

Kinetic study of quinoline analogs in liver cytosolic fractions from different species

Each triazolopyridine analog $(0.23-60 \mu \mathrm{M})$ was incubated with liver cytosolic fractions $(0.1$ $1 \mathrm{mg} / \mathrm{ml}$ ) for $30 \mathrm{~min}$.

\begin{tabular}{lcccc}
\hline Species & Test Compounds & $\begin{array}{c}V_{\text {max }} \\
\text { pmol/mg per minute }\end{array}$ & $\begin{array}{c}K_{\mathrm{m}} \\
\mu \mathrm{M}\end{array}$ & $\begin{array}{c}V_{\text {max }} / K_{\mathrm{m}} \\
\mu \mathrm{l} / \mathrm{min} \text { per milligram }\end{array}$ \\
\hline \multirow{2}{*}{ Mouse } & 63 & 14 & 38 & 0.36 \\
& 53 & 164 & 2.4 & 69 \\
& 51 & 25 & 1.0 & 25 \\
& 11 & 22 & 2.4 & 9.2 \\
Rat & 71 & 28 & 1.7 & 16 \\
& 63 & 1.8 & 64 & 0.03 \\
& 53 & 22 & 5.5 & 4.0 \\
& 51 & 19 & 1.1 & 17 \\
Monkey & 11 & 33 & 4.0 & 8.1 \\
& 71 & 11 & 1.0 & 11 \\
& 63 & 7.1 & 64 & 0.11 \\
& 53 & 95 & 2.7 & 27 \\
& 51 & 39 & 4.3 & 22 \\
Human & 11 & 229 & 5.9 & 2.0 \\
& 71 & 18 & 84 & 39 \\
& 63 & 23 & 2.6 & 0.22 \\
& 53 & 5.0 & 8.6 & 0.58 \\
& 51 & 14 & 63 & 0.23 \\
& 11 & 74 & 22 & 3.4 \\
\hline
\end{tabular}

AO-mediated metabolism can also occur in the heterocyclic core moiety in addition to the quinoline for some c-Met inhibitors. For example, the AO M formed on the heterocyclic core moiety instead of the quinoline was the major $\mathrm{M}$ for capmatinib (Xu et al., 2017; Dick, 2018). Similarly, AO oxidation exclusively on the imidazole core moiety was observed for c-Met inhibitor compound 1 (Zhao et al.,
2017). Many of our own c-Met inhibitors with $t_{1 / 2}$ values $<3$ minutes in cytosolic incubations were also mainly metabolized by $\mathrm{AO}$ on the heterocyclic core moiety instead of the 6-linked quinolines (unpublished data; Fig. 5). Like compound 71, EDGs such as O- and NH-linked cyclopropylmethyl moiety (examples 1 and 2; unpublished data; Fig. 5) were observed for these compounds that were unstable in cytosol. In addition to the aromatic aza-heterocycle with a vacant $\mathrm{C}-\mathrm{H}$ bond adjacent to a nitrogen atom (Pryde et al., 2010), it seems that an EDG ( $N$, O, phenyl) nearby would make the heterocycle core ring more vulnerable to AO metabolism. Several AO substrates with similar substructures from literature are listed in Fig. 5. The meta-O-linked EDGs in XK-469 (2-[4-(7-chloroquinoxalin-2-yl) oxyphenoxy]propanoate) (Anderson et al., 2005; Hutzler et al., 2012) and VU0409106 [3-fluoro- $N$-(4-methyl-2-thiazolyl)-5-(5-pyrimidinyloxy) benzamide] (Morrison et al., 2012), or the meta-N-linked EDG in example 2, and compounds 20 (Lepri et al., 2017; Cruciani et al., 2018) and 30 (Glatthar et al., 2016) made these compounds more susceptible to AO metabolism (Fig. 5). Based on the examples from literature and those we encountered in our internal c-Met program, a structural feature with potentially high AO liability was proposed as follows: 1) aza-heterocycle; 2) $o$-vacant $\mathrm{C}-\mathrm{H}$ bond; 3 ) $m$-small EDG (e.g., N, O, phenyl) (Fig. 5). It should be noted that this substructure is not automatically translated to AO substrate, since steric hindrance also plays a major role, as observed in this study.

In summary, the EDG and steric hindrance affect AO-mediated metabolism. For the triazolopyridine series of c-Met inhibitors, an unsubstituted quinoline is a poor AO substrate, and small substituents of EDG at the 3-quinoline increase the $\mathrm{AO}$ metabolism rate by decreasing the $\Delta \mathrm{G}$, while large substituents impede AO metabolism as a result of steric hindrance.<smiles>Cn1cc(-c2ccc3nnc(Sc4ccc5ncc(NC6COC6)cc5c4)n3c2)cn1</smiles><smiles>[R4][R4]([R])([H])Oc1ccc(OC(C)C(=O)O)cc1</smiles><smiles>Cc1cc(OC2CC2)cnc1C</smiles>

Unpublished example 1<smiles>Cc1cc(NC2CC2)cnc1C</smiles>

Unpublished example 2<smiles>CC(CN1CCOCC1)Nc1nc2ccc(-n3cnc4cnc5cnc(-c6ccncc6)cc5c43)cc2s1</smiles>

Example 30 Glatthar et al., 2016<smiles>c1cnc(NCCNc2cnc3ccccc3n2)nc1</smiles>

Example 2

Cruciani et al., 2018<smiles>Fc1cccnc1</smiles>

Proposed structure feature with high AO liability

1) aza-heterocycle

2) o-vacant $\mathrm{C}-\mathrm{H}$

3) $m$-smal EDG (O, N, phenyl, etc)

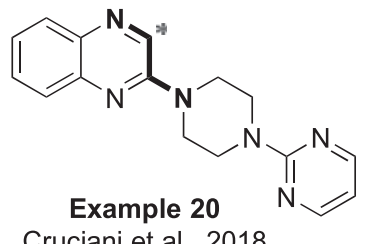

Fig. 5. Proposed common structural feature with high AO liability based on AO substrates from our internal c-Met program and the literature. Asterisks indicate an AO metabolism site. 


\section{Acknowledgments}

We thank Novartis c-Met Project team members, especially Global Discovery Chemistry Shanghai colleagues, for contributions to the study. We also thank Jane W. Zhang from Yale University (New Haven, CT) for proofreading the manuscript.

\section{Authorship Contributions}

Participated in research design: J. W. Zhang and J. Y. Zhang.

Conducted experiments: J. W. Zhang, Xiao, and Gao.

Contributed new reagents or analytic tools: J. W. Zhang, Xiao, Gao, and Yu.

Performed data analysis: J. W. Zhang, Xiao, Gao, Yu, and J. Y. Zhang.

Wrote or contributed to the writing of the manuscript: J. W. Zhang and J. Y. Zhang.

\section{References}

Anderson LW, Collins JM, Klecker RW, Katki AG, Parchment RE, Boinpally RR, LoRusso PM, and Ivy SP (2005) Metabolic profile of XK469 (2(R)-[4-(7-chloro-2-quinoxalinyl)oxyphenoxy]propionic acid; NSC698215) in patients and in vitro: low potential for active or toxic metabolite or for drug-drug interactions. Cancer Chemother Pharmacol 56:351-357.

Austin NE, Baldwin SJ, Cutler L, Deeks N, Kelly PJ, Nash M, Shardlow CE, Stemp G, Thewlis K, Ayrton A, et al. (2001) Pharmacokinetics of the novel, high-affinity and selective dopamine D3 receptor antagonist SB-277011 in rat, dog and monkey: in vitro/in vivo correlation and the role of aldehyde oxidase. Xenobiotica 31:677-686.

Cañadas I, Rojo F, Arumí-Uría M, Rovira A, Albanell J, and Arriola E (2010) C-MET as a new therapeutic target for the development of novel anticancer drugs. Clin Transl Oncol 12: 253-260.

Chen C, Deng H, Guo H, He F, Jiang L, Liang F, Mi Y, Wan H, Xu Y-C, Yu H, et al. (2013) inventors, Novartis AG, assignee. 6-substituted 3-(quinolin-6-ylthio)-[1,2,4] triazolo [4,3-a] pyradines as tyrosine kinase. PCT China WO/2013/038362, $2014 \mathrm{Jul} 23$.

Coelho C, Foti A, Hartmann T, Santos-Silva T, Leimkühler S, and Romão MJ (2015) Structural insights into xenobiotic and inhibitor binding to human aldehyde oxidase. Nat Chem Biol 11: 779-783.

Cruciani G, Milani N, Benedetti P, Lepri S, Cesarini L, Baroni M, Spyrakis F, Tortorella S, Mosconi E, and Goracci L (2018) From experiments to a fast easy-to-use computational methodology to predict human aldehyde oxidase selectivity and metabolic reactions. $J$ Med Chem 61:360-371.

Cui JJ (2014) Targeting receptor tyrosine kinase MET in cancer: small molecule inhibitors and clinical progress. J Med Chem 57:4427-4453.

Cui JJ, Shen H, Tran-Dubé M, Nambu M, McTigue M, Grodsky N, Ryan K, Yamazaki S, Aguirre S, Parker M, et al. (2013) Lessons from (S)-6-(1-(6-(1-methyl-1H-pyrazol-4-yl)-[1,2,4]triazolo[4,3-b]pyridazin-3-yl)ethyl)quinoline (PF-04254644), an inhibitor of receptor tyrosine kinase c-Met with high protein kinase selectivity but broad phosphodiesterase family inhibition leading to myocardial degeneration in rats. J Med Chem 56:6651-6665.

Dalvie D, Sun H, Xiang C, Hu Q, Jiang Y, and Kang P (2012) Effect of structural variation on aldehyde oxidase-catalyzed oxidation of zoniporide. Drug Metab Dispos 40:1575-1587.

Diamond S, Boer J, Maduskuie TP Jr, Falahatpisheh N, Li Y, and Yeleswaram S (2010) Speciesspecific metabolism of SGX523 by aldehyde oxidase and the toxicological implications. Drug Metab Dispos 38:1277-1285.

Dick RA (2018) Refinement of in vitro methods for identification of aldehyde oxidase substrates reveals metabolites of kinase inhibitors. Drug Metab Dispos 46:846-859.

Glatthar R, Stojanovic A, Troxler T, Mattes H, Möbitz H, Beerli R, Blanz J, Gassmann E, Drückes P, Fendrich G, et al. (2016) Discovery of imidazoquinolines as a novel class of potent, selective, and in vivo efficacious Cancer Osaka thyroid (COT) kinase inhibitors. J Med Chem 59: 7544-7560.

Hutzler JM, Yang Y-S, Albaugh D, Fullenwider CL, Schmenk J, and Fisher MB (2012) Characterization of aldehyde oxidase enzyme activity in cryopreserved human hepatocytes. Drug Metab Dispos 40:267-275.
Jia H, Dai G, Weng J, Zhang Z, Wang Q, Zhou F, Jiao L, Cui Y, Ren Y, Fan S, et al. (2014) Discovery of (S)-1-(1-(Imidazo[1,2-a]pyridin-6-yl)ethyl)-6-(1-methyl-1H-pyrazol-4-yl)-1H$[1,2,3]$ triazolo[4,5-b]pyrazine (volitinib) as a highly potent and selective mesenchymal-epithelia transition factor (c-Met) inhibitor in clinical development for treatment of cancer. J Med Chem 57:7577-7589.

Johns DG (1967) Human liver aldehyde oxidase: differential inhibition of oxidation of charged and uncharged substrates. J Clin Invest 46:1492-1505.

Jones JP and Korzekwa KR (2013) Predicting intrinsic clearance for drugs and drug candidates metabolized by aldehyde oxidase. Mol Pharm 10:1262-1268.

Kitamura S, Sugihara K, and Ohta S (2006) Drug-metabolizing ability of molybdenum hydroxylases. Drug Metab Pharmacokinet 21:83-98.

Lepri S, Ceccarelli M, Milani N, Tortorella S, Cucco A, Valeri A, Goracci L, Brink A, and Cruciani G (2017) Structure-metabolism relationships in human-AOX: chemical insights from a large database of aza-aromatic and amide compounds. Proc Natl Acad Sci USA 114:E3178-E3187.

Liu X, Newton RC, and Scherle PA (2010) Developing c-MET pathway inhibitors for cancer therapy: progress and challenges. Trends $\mathrm{Mol} \mathrm{Med} \mathrm{16:37-45.}$

Lolkema MP, Bohets HH, Arkenau H-T, Lampo A, Barale E, de Jonge MJA, van Doorn L, Hellemans P, de Bono JS, and Eskens FALM (2015) The c-Met tyrosine kinase inhibitor JNJ38877605 causes renal toxicity through species-specific insoluble metabolite formation. Clin Cancer Res 21:2297-2304.

Massey V, Komai H, Palmer G, and Elion GB (1970) On the mechanism of inactivation of xanthine oxidase by allopurinol and other pyrazolo[3,4-d]pyrimidines. J Biol Chem 245:2837-2844.

Morrison RD, Blobaum AL, Byers FW, Santomango TS, Bridges TM, Stec D, Brewer KA Sanchez-Ponce R, Corlew MM, Rush R, et al. (2012) The role of aldehyde oxidase and xanthine oxidase in the biotransformation of a novel negative allosteric modulator of metabotropic glutamate receptor subtype 5. Drug Metab Dispos 40:1834-1845.

Obach RS (2004) Potent inhibition of human liver aldehyde oxidase by raloxifene. Drug Metab Dispos 32:89-97.

Parikh PK and Ghate MD (2018) Recent advances in the discovery of small molecule c-Met kinase inhibitors. Eur J Med Chem 143:1103-1138.

Pryde DC, Dalvie D, Hu Q, Jones P, Obach RS, and Tran TD (2010) Aldehyde oxidase: an enzyme of emerging importance in drug discovery. J Med Chem 53:8441-8460.

Ryu JW, Han S-Y, Yun JI, Choi S-U, Jung H, Ha JD, Cho SY, Lee CO, Kang NS, Koh JS, et al (2011) Design and synthesis of triazolopyridazines substituted with methylisoquinolinone as selective c-Met kinase inhibitors. Bioorg Med Chem Lett 21:7185-7188.

Sahi J, Khan KK, and Black CB (2008) Aldehyde oxidase activity and inhibition in hepatocytes and cytosolic fractions from mouse, rat, monkey and human. Drug Metab Lett 2:176-183.

Stiefl N, Gedeck P, Chin D, Hunt P, Lindvall M, Spiegel K, Springer C, Biller S, Buenemann C, Kanazawa T, et al. (2015) FOCUS--development of a global communication and modeling platform for applied and computational medicinal chemists. J Chem Inf Model 55:896-908.

Torres RA, Korzekwa KR, McMasters DR, Fandozzi CM, and Jones JP (2007) Use of density functional calculations to predict the regioselectivity of drugs and molecules metabolized by aldehyde oxidase. $J$ Med Chem 50:4642-4647.

Xu Y, Li L, Wang Y, Xing J, Zhou L, Zhong D, Luo X, Jiang H, Chen K, Zheng M, et al. (2017) Aldehyde oxidase mediated metabolism in drug-like molecules: a combined computational and experimental study. J Med Chem 60:2973-2982.

Zhao F, Zhang LD, Hao Y, Chen N, Bai R, Wang YJ, Zhang CC, Li GS, Hao LJ, Shi C, et al. (2017) Identification of 3-substituted-6-(1-(1H-[1,2,3]triazolo[4,5-b]pyrazin-1-yl)ethyl)quinoline derivatives as highly potent and selective mesenchymal-epithelial transition factor (c-Met) inhibitors via metabolite profiling-based structural optimization. Eur J Med Chem 134:147-158.

Zientek M, Jiang Y, Youdim K, and Obach RS (2010) In vitro-in vivo correlation for intrinsic clearance for drugs metabolized by human aldehyde oxidase. Drug Metab Dispos 38: $1322-1327$.

Address correspondence to: Jiang Wei Zhang, Novartis Institutes for BioMedica Research, F2B4, Novartis Campus, No. 4218 Jinke Road, Shanghai 201203, People's Republic of China. E-mail: jiangwei.zhang@novartis.com; or Ji Yue (Jeff) Zhang, Novartis Institutes for BioMedical Research, F2B4, Novartis Campus, No.4218 Jinke Road, Shanghai 201203, People's Republic of China. E-mail: jeff. zhang@novartis.com 